PROCEEDINGS OF THE

AMERICAN MATHEMATICAL SOCIETY

Volume 137, Number 10, October 2009, Pages 3547-3552

S 0002-9939(09)09920-1

Article electronically published on May 27, 2009

\title{
AN OBSTRUCTION TO A KNOT BEING DEFORM-SPUN VIA ALEXANDER POLYNOMIALS
}

\author{
RYAN BUDNEY AND ALEXANDRA MOZGOVA
}

(Communicated by Daniel Ruberman)

\begin{abstract}
We show that if a co-dimension two knot is deform-spun from a lower-dimensional co-dimension 2 knot, there are constraints on the Alexander polynomials. In particular this shows, for all $n$, that not all co-dimension 2 knots in $S^{n}$ are deform-spun from knots in $S^{n-1}$.
\end{abstract}

In co-dimension 2 knot theory [6], typically the term ' $n$-knot' denotes a manifold pair $\left(S^{n+2}, K\right)$ where $K$ is the image of a smooth embedding $f: S^{n} \rightarrow S^{n+2}$. An $n$-ball pair is a pair $\left(D^{n+2}, J\right)$ where $J$ is the image of a smooth embedding $f: D^{n} \rightarrow D^{n+2}$ such that $f^{-1}\left(\partial D^{n+2}\right)=\partial D^{n}$. Every $n$-knot $K$ is isotopic to a union $\left(S^{n+2}, K\right)=\left(D^{n+2}, J\right) \cup_{\partial}\left(D^{n+2}, D^{n}\right)$ for some unique isotopy class of $n$-ball pair $\left(D^{n+2}, J\right)$, provided we consider $K$ to be oriented. Let $\operatorname{Diff}\left(D^{n+2}, J\right)$ denote the group of diffeomorphisms of an $n$-ball pair $\left(D^{n+2}, J\right)$. That is, $f \in$ $\operatorname{Diff}\left(D^{n+2}, J\right)$ means that $f$ is a diffeomorphism of $D^{n+2}$ which restricts to the identity on $\partial D^{n+2}=S^{n+1}$, is isotopic to the identity (rel boundary) as a diffeomorphism of $D^{n+1}$, and $f$ preserves $J, f(J)=J$. We say an $n$-knot $\left(S^{n+2}, K\right)$ is deformspun from an $(n-1)$-knot $\left(S^{n+1}, K^{\prime}\right)=\left(D^{n+1}, J^{\prime}\right) \cup_{\partial}\left(D^{n+1}, D^{n-1}\right)$ if there exists $g \in \operatorname{Diff}\left(D^{n+1}, J^{\prime}\right)$ such that the pair $\left(\left(D^{n+1}, J^{\prime}\right) \times{ }_{g} S^{1}\right) \cup_{\partial}\left(\left(S^{n}, S^{n-1}\right) \times D^{2}\right)$ is diffeomorphic to the pair $\left(S^{n+2}, K\right)$. Here $\left(D^{n+1}, J^{\prime}\right) \times{ }_{g} S^{1}$ is the bundle over $S^{1}$ with fibre $\left(D^{n+1}, J^{\prime}\right)$ and monodromy given by $g$ (i.e., $\left(D^{n+1}, J^{\prime}\right) \times{ }_{g} S^{1}=$ $\left(\left(D^{n+1}, J^{\prime}\right) \times \mathbb{R}\right) / \mathbb{Z}$, where $\mathbb{Z}$ acts diagonally) on $\left(D^{n+1}, J^{\prime}\right)$ and as the group of universal covering transformations for $\mathbb{R} \rightarrow S^{1}$.

To picture a deform-spun knot, let $g_{t}$ be a null-isotopy of $g$; i.e., $g_{0}=g, g_{1}=$ $I d_{D^{n+1}}$ and $g_{t}$ is a diffeomorphism of $D^{n+1}$ which restricts to the identity on $\partial D^{n+2}$ for all $0 \leq t \leq 1$. Consider $S^{n+2}$ to be the union of a great $n$-sphere $S^{n}$ and a disjoint trivial vector bundle over $S^{1}$. Identify this trivial vector bundle over $S^{1}$ with $S^{1} \times \operatorname{int}\left(D^{n+1}\right)$ and identify $S^{1}$ with $\mathbb{R} / \mathbb{Z}$. We assume that the inclusion $S^{1} \times \operatorname{int}\left(D^{n+1}\right) \rightarrow S^{n+2}$ extends to a map $S^{1} \times D^{n+1} \rightarrow S^{n+2}$ such that the restriction $S^{1} \times S^{n} \rightarrow S^{n+2}$ factors as projection onto the great sphere $S^{n}$ followed

Received by the editors November 18, 2007, and, in revised form, February 16, 2009.

2000 Mathematics Subject Classification. Primary 57R40.

The authors would like to thank the Max Planck Institute for Mathematics for its hospitality. The first author would also like to thank the Institut des Hautes Études Scientifiques for its hospitality, as well as Danny Ruberman and an anonymous referee for many useful comments on the paper. 


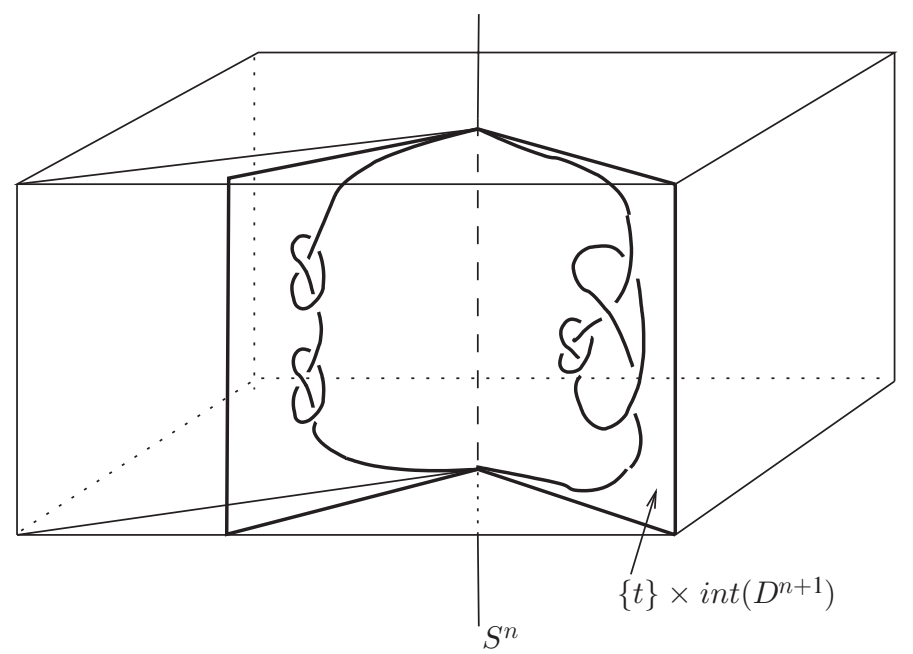

FiguRE 1

by the inclusion $S^{n} \rightarrow S^{n+2}$. Then the set $\left\{(t, x) \in S^{1} \times \operatorname{int}\left(D^{n+1}\right): x=g_{t}(p), p \in\right.$ $\left.\operatorname{int}\left(J^{\prime}\right)\right\}$ is a subset of $S^{n+2}$ whose closure is an $n$-knot. This is the deform-spun knot; see Figure 1.

The main observation of this paper is that if $K$ is an $n$-knot, deform-spun from an $(n-1)$-knot $K^{\prime}$, then there is a relationship between the Alexander modules of $K$ and $K^{\prime}$ which give rise to constraints on the Alexander polynomials $\Delta_{1}, \cdots, \Delta_{n}$ of $K$.

Theorem 0.1. Let $K$ be an $n$-knot which is deform-spun. Then there exist polynomials $q_{i} \in \Lambda=\mathbb{Q}\left[t^{ \pm 1}\right]=\mathbb{Q}[\mathbb{Z}]$ for $i=0,1, \cdots, n$ which satisfy $q_{i+1} q_{i}=\Delta_{i+1}$ $\left(q_{0}=q_{n}=1\right)$ and $q_{n-i}=\overline{q_{i}}$ for all $i$, where we use the convention $\overline{q_{i}}(t)=q_{i}\left(t^{-1}\right)$.

An elementary consequence of this theorem is that for each $n \geq 2$, not every $n$ knot is deform-spun from an $(n-1)$-knot. This follows from the work of Levine [4] who gave a characterization of the Alexander modules of co-dimension 2-knots. In particular Levine shows that an $n$-knot has Alexander polynomials $\Delta_{1}, \cdots, \Delta_{n} \in \Lambda$ which satisfy the relations $\Delta_{i}(1) \neq 0, \overline{\Delta_{i}}=\Delta_{n-i}$ for all $i$. Moreover, these relations are complete in the sense that given any $n$ polynomials which satisfy these relations, there is an $n$-knot which has the specified Alexander polynomials. The case $n=2$ has a particularly simple example. Theorem 0.1 states that if $K$ is deform-spun, then $\overline{\Delta_{1}}=\Delta_{1}$, yet there are 2 -knots such that $\Delta_{1}$ is not symmetric. See example 10 of Fox's Quick Trip [2], which describes a 2-knot such that $\Delta_{1}(t)=2 t-1$.

Litherland's deform-spinning construction has its origin in papers of Fox and Zeeman. Fox's 'Rolling' 3] paper gave a heuristic outline of the notion eventually called deform-spinning, as a graphing process from a 'relative 2-dimensional braid group' which nowadays is frequently called the fundamental group of the space of knots, or (in a slightly different setting) the mapping class group of the knot complement [1. Zeeman proved that the complements of co-dimension two $n$-twistspun knots fibre over $S^{1}$ provided $n \neq 0$ [8]. Litherland [7] went on to formulate a general situation where a deform-spun knot complements fibre over $S^{1}$. Specifically, Litherland proved that if the diffeomorphism $g:\left(D^{n+1}, J^{\prime}\right) \rightarrow\left(D^{n+1}, J^{\prime}\right)$ preserves 
a Seifert surface for the knot $\left(S^{n+1}, K^{\prime}\right)$ corresponding to the $(n-1)$-disc pair $\left(D^{n+1}, J^{\prime}\right)$, then the deform-spun knot associated to the diffeomorphism $M \circ g$ : $\left(D^{n+1}, J^{\prime}\right) \rightarrow\left(D^{n+1}, J^{\prime}\right)$ has a complement which fibres over $S^{1}$, provided $M$ : $\left(D^{n+1}, J^{\prime}\right) \rightarrow\left(D^{n+1}, J^{\prime}\right)$ is a non-zero power of the meridional Dehn twist about $J^{\prime}$.

This paper was largely motivated by a result in 'high' co-dimension knot theory. In the paper 1 the first author gave a new proof of Haefliger's theorem, where the monoid of isotopy classes of smooth embeddings of $S^{j}$ in $S^{n}$ is a group, provided $n-j>2$. The heart of the proof is showing that if $n-j>2$, then every knot $\left(S^{n}, K\right)$ (where $K \simeq S^{j}$ ) is deform-spun from a lower-dimensional knot $\left(S^{n-1}, K^{\prime}\right)$, where $K^{\prime} \simeq S^{j-1}$. Moreover, all knots $\left(S^{n}, K\right)$ are $i$-fold deform-spun for $i=2(n-j)-4$, in the sense that one obtains $\left(S^{n}, K\right)$ to be iterating the deform-spinning process $i$ times. So in a sense this paper represents an investigation of the extreme case $n-j=2$. A second motivation is the observation that frequently the groups $\pi_{0} \operatorname{Diff}\left(D^{3}, J^{\prime}\right)\left(\left(D^{3}, J^{\prime}\right)\right.$ a 1-ball pair $)$ are quite large [1], in the sense that their classifying spaces all have the homotopy-type of finite-dimensional manifolds, but the dimension of these manifolds can be arbitrarily large. So there are many ways to construct 2-knots by deform-spinning a 1-knot. As far as the authors know, this paper represents the first-known obstructions to knots being deform-spun.

\section{Asymmetry obstruction}

Given a co-dimension 2-knot $K$ in $S^{n+2}$, the complement of the knot, $C_{K}$, is a homology $S^{1}$. Let $\tilde{C}_{K}$ denote the universal abelian cover of $C_{K}$, i.e., the cover corresponding to the kernel of the abelianization map $\pi_{1} C_{K} \rightarrow \mathbb{Z}$, and consider $H_{i}\left(\tilde{C}_{K} ; \mathbb{Q}\right)$ to be a module over the group-ring of covering transformations $\Lambda=$ $\mathbb{Q}[\mathbb{Z}]=\mathbb{Q}\left[t, t^{-1}\right]$. This is called the $i$-th Alexander module of $K . H_{i}\left(\tilde{C}_{K} ; \mathbb{Q}\right)$ is a finitely-generated torsion $\Lambda$-module 4 for each $i$, so $H_{i}\left(\tilde{C}_{K} ; \mathbb{Q}\right) \simeq \bigoplus_{j} \Lambda / p_{j}$ for some collection of polynomials $p_{j}$. The product of these polynomials $\prod_{j} p_{j}$ is called the $i$-th Alexander polynomial of $K$, or the order ideal of the $i$-th Alexander module $H_{i}\left(\tilde{C}_{K} ; \mathbb{Q}\right)$, denoted $\Delta_{i}$. In general, the order ideal of a finitely generated torsion $\Lambda$-module $M$ will be denoted $\Delta_{M}$. A theorem of Levine's [4] is that Poincaré Duality combined with the Universal Coefficient Theorem induces an isomorphism $\overline{H_{i}\left(\tilde{C}_{K} ; \mathbb{Q}\right)} \simeq \operatorname{Ext}_{\Lambda}\left(H_{n+1-i}\left(\tilde{C}_{K} ; \mathbb{Q}\right), \Lambda\right)$. Here, if $M$ is a $\Lambda$-module, $\bar{M}$ denotes the conjugate $\Lambda$-module. This is a module whose underlying $\mathbb{Q}$-vector space is $M$, but where action of the generator $t$ on $\bar{M}$ is defined as the action of $t^{-1}$ on $M$. Thus, the only Alexander polynomials of $K$ which can be non-trivial are $\Delta_{1}, \cdots, \Delta_{n}$, and they satisfy the relation $\overline{\Delta_{i}}=\Delta_{n+1-i}$ for all $i$.

We collect some elementary results about $\Lambda$-modules that will be of use in the proof of Theorem 0.1 . To state the lemma, let $\mathbb{Q}(\Lambda)$ denote the field of fractions of $\Lambda$, i.e., the field which consists of rational Laurent polynomials.

Lemma 1.1. (a) (see [6], 7.2.7) Given a short exact sequence of finitely generated torsion $\Lambda$-modules

$$
0 \rightarrow H_{1} \rightarrow H \rightarrow H_{2} \rightarrow 0
$$

the order ideals satisfy $\Delta_{H_{1}} \Delta_{H_{2}}=\Delta_{H}$. 
(b) (see 4], Proposition 4.1) Let $H$ be a finitely-generated torsion $\Lambda$-module. There is a natural isomorphism of $\Lambda$-modules

$$
\operatorname{Ext}_{\Lambda}(H, \Lambda) \simeq \operatorname{Hom}_{\Lambda}(H, \mathbb{Q}(\Lambda) / \Lambda) .
$$

(c) With the same setup as (b), there is a natural isomorphism of $\mathbb{Q}$-vector spaces

$$
\operatorname{Hom}_{\Lambda}(H, \mathbb{Q}(\Lambda) / \Lambda) \simeq \operatorname{Hom}_{\mathbb{Q}}(H, \mathbb{Q})
$$

where we interpret $\Lambda \subset \mathbb{Q}(\Lambda)$ as the rational Laurent polynomials with denominator 1 .

(d) Let $g: H \rightarrow H$ be a $\Lambda$-linear map, where $H$ is a finitely-generated torsion $\Lambda$-module. Let $g^{*}: \operatorname{Ext}_{\Lambda}(H, \Lambda) \rightarrow \operatorname{Ext}_{\Lambda}(H, \Lambda)$ be the Ext-dual of $g$. Then $\operatorname{ker}(g)$ and $\operatorname{ker}\left(g^{*}\right)$ have the same order ideals.

Proof of item (c). Consider a rational polynomial $\frac{p}{q} \in \mathbb{Q}(\Lambda)$. The division algorithm allows us to write $p=s q+r$ for Laurent polynomials $s, r \in \Lambda$, where $r \in \mathbb{Q}[t]$ and $\operatorname{deg}(r)<\operatorname{deg}(q)$. To ensure that $r$ is unique, we demand that $G C D(p, q)=1$, $q \in \mathbb{Q}[t]$ and the constant coefficient of $q$ is 1 . Define a function $\mathbb{Q}(\Lambda) / \Lambda \rightarrow \mathbb{Q}$ by sending $\frac{p}{q}$ to the constant coefficient of $r$. The composition with this map is a $\mathbb{Q}$-linear homomorphism, $\operatorname{Hom}_{\Lambda}(H, \mathbb{Q}(\Lambda) / \Lambda) \rightarrow \operatorname{Hom}_{\mathbb{Q}}(H, \mathbb{Q})$, which is natural and respects connect-sum decompositions of the domain $H$. Thus to verify that it is an isomorphism, we need only check it on a torsion $\Lambda$-module with one generator:

$$
\operatorname{Hom}_{\Lambda}(\Lambda / p, \mathbb{Q}(\Lambda) / \Lambda) \rightarrow \operatorname{Hom}_{\mathbb{Q}}(\Lambda / p, \mathbb{Q}) .
$$

In this case the target space has dimension $\operatorname{deg}(p)$, the basis given by the dual basis to the polynomials $t^{i}$ for $0 \leq i<\operatorname{deg}(p)$. The domain also has dimension $\operatorname{deg}(p)$, with basis given by homomorphisms that send 1 to $t^{i} / p$ where $0 \leq i<\operatorname{deg}(p)$. Hence the map is a bijection between these basis vectors.

To prove item (d), consider the 'prime factorization' of $H$. Let $P \subset \Lambda$ be the prime factors of the order ideal $\Delta_{H}$. Given $p \in P$ let $H_{p} \subset H$ be the sub-module of elements of $H$ killed by a power of $p$; thus $\bigoplus_{p \in P} H_{p} \simeq H$. Since $g$ must respect the splitting, we have maps $g_{p}$ such that

$$
g=\bigoplus_{p \in P} g_{p}: H_{p} \rightarrow H_{p} .
$$

Thus,

$$
\Delta_{k e r(g)}=\prod_{p \in P} \Delta_{k e r\left(g_{p}\right)} .
$$

Let $d_{p} \in \mathbb{Z}$ be defined so that $\Delta_{k e r\left(g_{p}\right)}=p^{d_{p}}$. By part (c), $g$ and $g^{*}$ can be thought of as the $\operatorname{Hom}_{\mathbb{Q}}(\cdot, \mathbb{Q})$-duals of each other. Thus $\operatorname{ker}(g)$ and $\operatorname{ker}\left(g^{*}\right)$ have the same dimension as $\mathbb{Q}$-vector spaces, and so $\operatorname{dim}_{\mathbb{Q}}\left(\operatorname{ker}\left(g_{p}\right)\right)=\operatorname{deg}(p) d_{p}$ and $\Delta_{k e r\left(g_{p}\right)}$ is determined by the rank of $\operatorname{ker}\left(g_{p}\right)$ as a $\mathbb{Q}$-vector space. Hence $\operatorname{ker}(g)$ and $\operatorname{ker}\left(g^{*}\right)$ have the same order ideals.

Remark. Although they have the same order ideals, in general the two kernels are not isomorphic as $\Lambda$-modules. An example is given by $g: \Lambda / p \oplus \Lambda / p^{2} \rightarrow \Lambda / p \oplus \Lambda / p^{2}$ defined by $g(a, b)=(0, p a)$. In this case, $\operatorname{ker}(g) \simeq \Lambda / p^{2}$, while $\operatorname{ker}\left(g^{*}\right) \simeq \bigoplus_{2} \Lambda / p$.

Proof of of Theorem 0.1 . Let $C_{K}$ be the complement of an open tubular neighbourhood of $K \subset S^{n+2}$, and let $C_{K^{\prime}}$ be the complement of an open tubular neighbourhood of $K^{\prime} \subset S^{n+1}$. As in the introduction, let $g:\left(D^{n+1}, J^{\prime}\right) \rightarrow\left(D^{n+1}, J^{\prime}\right)$ 
be the diffeomorphism for the deform-spinning construction of $K$ from $K^{\prime}$, so we can isotope $g$ so that it preserves a regular neighbourhood of $J^{\prime} \cup S^{n}$. Therefore $g$ restricts to a diffeomorphism of $C_{K^{\prime}}$ (which we can think of as the complement of an open regular neighbourhood of $S^{n} \cup J^{\prime}$ in $\left.D^{n+1}\right)$, giving a diffeomorphism

$$
C_{K} \simeq\left(C_{K^{\prime}} \times g S^{1}\right) \cup_{\nu S^{1} \times S^{1}}\left(\left(\nu S^{1}\right) \times D^{2}\right)
$$

where $\nu S^{1}$ is a trivial $D^{n-1}$-bundle over $S^{1}$ (a meridian of $\partial C_{K^{\prime}}$ ). The decomposition lifts to the universal abelian covering space, giving the isomorphism $H_{1}\left(\tilde{C}_{K} ; \mathbb{Q}\right)$ $\simeq \operatorname{coker}\left(I-g_{1 *}\right)$ and short exact sequences

$$
0 \rightarrow \operatorname{coker}\left(g_{i *}-I\right) \rightarrow H_{i}\left(\tilde{C}_{K} ; \mathbb{Q}\right) \rightarrow \operatorname{ker}\left(g_{(i-1) *}-I\right) \rightarrow 0, \quad i>1,
$$

with $g_{i *}: H_{i}\left(\tilde{C}_{K^{\prime}} ; \mathbb{Q}\right) \rightarrow H_{i}\left(\tilde{C}_{K^{\prime}} ; \mathbb{Q}\right)$ the induced map coming from $\tilde{g}: \tilde{C}_{K^{\prime}} \rightarrow \tilde{C}_{K^{\prime}}$. Let $q_{i}$ be the order ideal of $\operatorname{coker}\left(g_{i *}-I\right)$.

The map $g_{i *}-I: H_{i}\left(\tilde{C}_{K^{\prime}} ; \mathbb{Q}\right) \rightarrow H_{i}\left(\tilde{C}_{K^{\prime}} ; \mathbb{Q}\right)$ gives rise to a canonical short exact sequence

$$
0 \rightarrow \operatorname{ker}\left(g_{i *}-I\right) \rightarrow H_{i}\left(\tilde{C}_{K^{\prime}} ; \mathbb{Q}\right) \rightarrow i m g\left(g_{i *}-I\right) \rightarrow 0
$$

and the inclusion $i m g\left(g_{i *}-I\right) \rightarrow H_{i}\left(\tilde{C}_{K^{\prime}} ; \mathbb{Q}\right)$ to another,

$$
0 \rightarrow i m g\left(g_{i *}-I\right) \rightarrow H_{i}\left(\tilde{C}_{K^{\prime}} ; \mathbb{Q}\right) \rightarrow \operatorname{coker}\left(g_{i *}-I\right) \rightarrow 0 .
$$

Lemma 1.1(a) applied to our short exact sequences tells us that $\Delta_{i}=q_{i} q_{i-1}$.

We now reconsider the proof of the symmetry of the Alexander polynomial of a knot in $S^{3}[5,6]$ or, more precisely, the isomorphism $\overline{H_{i}\left(\tilde{C}_{K^{\prime}} ; \mathbb{Q}\right)} \simeq H_{n-i}\left(\tilde{C}_{K^{\prime}} ; \mathbb{Q}\right)$ derived from Poincaré Duality [4, paying special attention to naturality with respect to diffeomorphisms $g \in \operatorname{Diff}\left(C_{K^{\prime}}\right)$, with an eye towards proving the symmetry conditions $\overline{q_{n-i}}=q_{i}$.

(1) $H_{i}\left(\tilde{C}_{K^{\prime}} ; \mathbb{Q}\right) \simeq H_{i}\left(\tilde{C}_{K^{\prime}}, \partial ; \mathbb{Q}\right)$ : This is a natural isomorphism coming from the long exact sequence of a pair.

(2) $H_{i}\left(\tilde{C}_{K^{\prime}}, \partial ; \mathbb{Q}\right) \simeq \overline{H^{n+1-i}\left(\tilde{C}_{K^{\prime}} ; \mathbb{Q}\right)}$ : This is the Poincaré duality isomorphism; it is also natural, although it reverses arrows [4].

(3) $H^{n+1-i}\left(\tilde{C}_{K^{\prime}} ; \mathbb{Q}\right) \simeq \operatorname{Ext}_{\Lambda}\left(H_{n-i}\left(\tilde{C}_{K^{\prime}} ; \mathbb{Q}\right), \Lambda\right)$ : This is a natural isomorphism coming from the universal coefficient theorem [4].

(4) $\operatorname{Ext}_{\Lambda}\left(H_{n-i}\left(\tilde{C}_{K^{\prime}} ; \mathbb{Q}\right), \Lambda\right) \simeq H_{n-i}\left(\tilde{C}_{K^{\prime}} ; \mathbb{Q}\right)$ : This last result uses the fact that both modules have a square presentation matrix, with one being the transpose of the other. Since $\Lambda$ is a principal ideal domain, the presentation matrices are equivalent to the same diagonal matrices. This isomorphism is not natural.

Thus we have a non-natural isomorphism $H_{i}\left(\tilde{C}_{K} ; \mathbb{Q}\right) \simeq \overline{H_{n-i}\left(\tilde{C}_{K} ; \mathbb{Q}\right)}$. The natural part of the isomorphism can be expressed by the commutative diagram

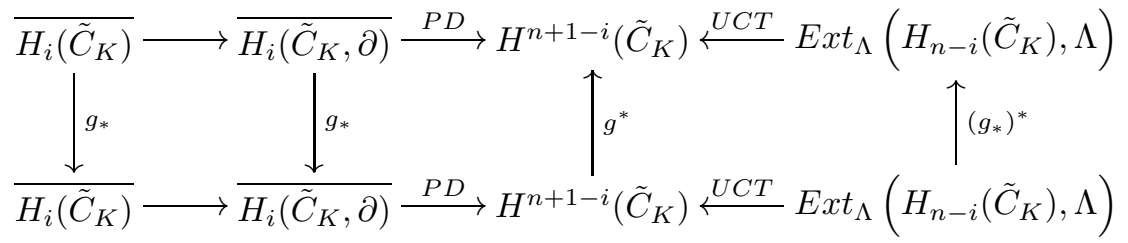

This gives us an isomorphism of $\Lambda$-modules $\overline{\operatorname{ker}\left(I-g_{i *}\right)} \simeq \operatorname{ker}\left(I-\left(g_{(n-i) *}^{-1}\right)^{*}\right)$, so

$$
\overline{\operatorname{ker}\left(I-g_{i *}\right)} \simeq \operatorname{ker}\left(I-\left(g_{(n-i) *}^{-1}\right)^{*}\right)=\operatorname{ker}\left(I-\left(g_{(n-i) *}\right)^{*}\right) \text {. }
$$


Lemma 1.1(d) tells us that $\operatorname{ker}\left(I-\left(g_{(n-i) *}\right)^{*}\right)$ and $\operatorname{ker}\left(I-g_{(n-i) *}\right)$ have the same order ideals. Thus, $\overline{q_{i}}=q_{n-i}$.

\section{Comments And Questions}

Levine [4 has a complete characterization of the Alexander modules of codimension two knots. A natural question would be, Could one further derive other obstructions to deform-spinning from the Alexander modules of knots? The primary aspect of Levine's work that we've neglected is the $\mathbb{Z}$-torsion submodule of $H_{i}\left(\tilde{C}_{K} ; \mathbb{Z}\right)$. Simple experiments show that when $K \subset S^{n+2}$ is deform-spun from a knot $K^{\prime} \subset S^{n+1}$, the Alexander modules of $K$ can have $\mathbb{Z}$-torsion, even when the Alexander modules of $K^{\prime}$ do not. Moreover, twist-spinning suffices to produce many such examples. So any torsion obstructions to deform-spinning, if they exist, would likely be fairly subtle.

In co-dimension larger than two, deform-spinning is the boundary map in the pseudo-isotopy long exact sequence for embedding spaces and diffeomorphism groups [1. Moreover, Cerf's Pseudoisotopy Theorem states that in the case of diffeomorphism groups of discs, this map is onto, provided the dimension of the disc is 6 or larger. So one might expect an analogy.

Question 2.1. Is there a simple characterization of deform-spun co-dimension two knots $K \subset S^{n+2}$ (provided $n$ is large)?

One would certainly expect more obstructions to deform-spinning than the ones in this paper. For example, let $K_{1}$ and $K_{2}$ be two otherwise unrelated 2-knots such that $\Delta_{K_{1}}(t)=2-t$ and $\Delta_{K_{2}}(t)=2 t-1$. Their connect sum has Alexander polynomial $\Delta_{K_{1} \# K_{2}}(t)=-2 t^{2}+3 t-2$, which is symmetric, but we have no reason to expect that $K_{1} \# K_{2}$ is deform-spun.

\section{REFERENCES}

1. R. Budney, A Family of Embedding Spaces, Geometry \& Topology Monographs, 13, 2007.

2. R.H. Fox, A quick trip through knot theory, in "Topology of 3-Manifolds and Related Topics", M.K. Fort (ed.), Prentice-Hall, Englewood Cliffs, NJ, 1962, pp. 120-167. MR0140099 $(25: 3522)$

3. R.H. Fox, Rolling, Bull. Amer. Math. Soc. 72 (1966), 162-164. MR0184221 (32:1694)

4. J. Levine, Knot modules. I, Trans. Amer. Math. Soc. 229 (1977), 1-50. MR0461518 (57:1503)

5. C. Gordon, Some aspects of classical knot theory, in "Knot Theory", Lecture Notes in Math., 685, Springer, Berlin, 1978, pp. 1-60. MR.521730 (80f:57002)

6. A. Kawauchi, A Survey of Knot Theory, Springer-Verlag, Tokyo, 1990. MR 1417494 (97k:57011)

7. R.A. Litherland, Deforming twist-spun knots, Trans. Amer. Math. Soc. 250 (1979), 311-331. MR.530058 (80i:57015)

8. E. Zeeman, Twisting spun knots, Trans. Amer. Math. Soc. 115 (1965), 471-495. MR0195085 $(33: 3290)$

Department of Mathematics and Statistics, University of Victoria, P.O. Box 3045 STN CSC, Victoria, British Columbia, Canada V8W 3P4

E-mail address: rybu@uvic.ca

ACRI, 260 route du Pin Montard, BP 234, F-06904 Sophia Antipolis Cedex, France

E-mail address: sasha.mozgova@gmail.com 